LA-UR-02-5359

Approved for public release; distribution is unlimited.

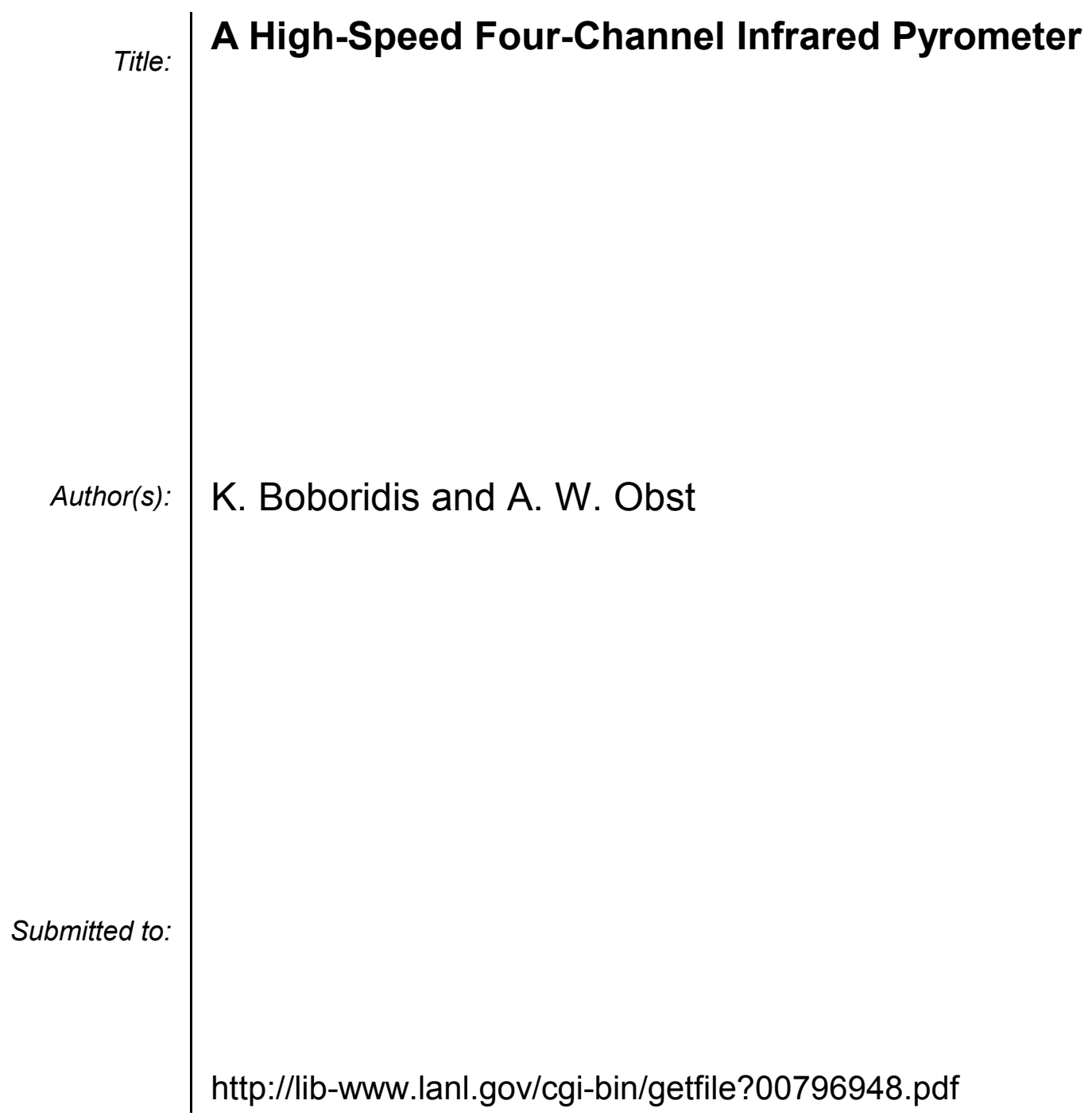

Los Alamos National Laboratory, an affirmative action/equal opportunity employer, is operated by the University of California for the U.S. Department of Energy under contract W-7405-ENG-36. By acceptance of this article, the publisher recognizes that the U.S. Government retains a nonexclusive, royaltyfree license to publish or reproduce the published form of this contribution, or to allow others to do so, for U.S. Government purposes. Los Alamos National Laboratory requests that the publisher identify this article as work performed under the auspices of the U.S. Department of Energy. Los Alamos National Laboratory strongly supports academic freedom and a researcher's right to publish; as an institution, however, the Laboratory does not endorse the viewpoint of a publication or guarantee its technical correctness. 


\title{
A High-Speed Four-Channel Infrared Pyrometer
}

\author{
K. Boboridis and A. W. Obst \\ Physics Division, MS H803, Los Alamos National Laboratory, Los Alamos, New Mexico 87545.
}

\begin{abstract}
A high-speed, four-wavelength pyrometer has been developed for dynamic temperature measurements on samples that are heated by shock compression. The pyrometer uses a pair of off-axis parabolic mirrors to collect radiance emitted from a target of $1 \mathrm{~mm}$ in diameter. A single optical fiber delivers the collected radiant flux to the detector housing. Three dichroic beam splitters are used to spectrally split the light into four beams that are then focused onto an equal number of $\mathrm{LN}_{2}$-cooled InSb photodetectors. Broad bandwidth interference filters that are nominally centered at 1.8, 2.4, 3.4, and $5.0 \mu \mathrm{m}$ define the wavelength ranges of the four channels. The blackbody-temperature threshold of the pyrometer is at about $400 \mathrm{~K}$. The signals are recorded at intervals as short as $20 \mathrm{~ns}$ using a four-channel digital oscilloscope. Procedures for calibration and temperature measurements are described.
\end{abstract}

\section{INTRODUCTION}

This paper describes a new high-speed, fourwavelength pyrometer that has been developed for dynamic temperature measurements on samples that are heated by shock compression. These experiments are a primary diagnostic in the determination of the equations of state (EOS) of materials, in which temperature is an important parameter. Shock compression is usually achieved with the use of high explosives (HE) or through the impact on the sample of a flyer plate that has been accelerated to high speeds. In both cases, the experiment duration is very short (a few microseconds at most) and the temperature may be varying rapidly, which is why optical pyrometry is well suited. The drawback of this technique, however, is that the sample emittance has to be known for the measurement to be accurate. Unfortunately, in the majority of cases the emittance of the samples as a function of temperature is unknown and may be changing during the experiments as a function of surface roughness, as well. Traditionally, pyrometers that detect radiance in more than one spectral band have been used to determine temperature in the absence of sufficient knowledge about the sample emittance. Numerous methods for inferring thermodynamic temperature from multiwavelength radiance temperature measurements have been developed and used in various situations [1-3].

\section{DESIGN OF THE PYROMETER}

The new pyrometer consists of two units that are linked with an optical fiber. The unit containing the front optics collects thermally emitted radiance from the sample and focuses it on the optical fiber that transmits it to the detector unit. There, the transmitted light is spectrally split into four beams and detected by the fast photodiodes. This design was primarily dictated by the difficulties presented by shock experiments, such as the great likelihood of severe damage to equipment that is placed in front of the sample, space limitations, and, in some cases, considerable levels of electromagnetic interference (EMI). In addition, this modular design offers flexibility in the choice of front optics that are most suitable for the particular experiment and makes the system more easily portable.

\section{Front Optics}

As shown in Fig. 1, the front optics consist of a pair of off-axis parabolic mirrors (OAP) that collect thermally emitted radiance from the sample and focus it on the optical fiber that transmits it to the detector unit. For aiming and focussing, a flip mirror can be rotated into the optical path to form an image of the sample in front of a pocket microscope with a precision reticle. 


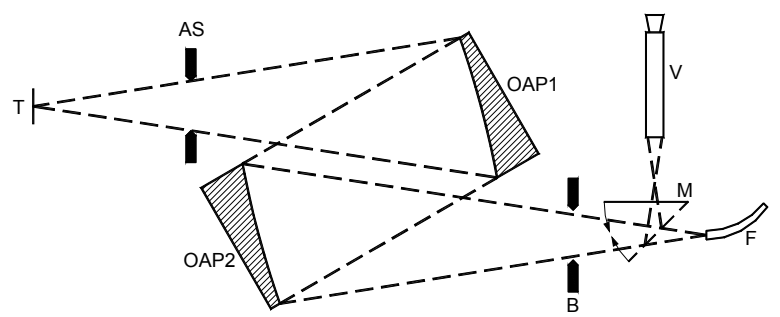

FIGURE 1. Schematic diagram of the front-optics unit of the four-wavelength infrared pyrometer. T, target; AS, aperture stop; OAP, off-axis parabolic mirror; B, baffle; M, flip mirror; V, viewing microscope; F, fiber.

The mirrors are diamond-turned, made of aluminum and coated with a protective silicon monoxide layer. They are designed to be used at an off-axis angle of $30^{\circ}$, have a diameter of $101.6 \mathrm{~mm}$, a parent focal length of $304.8 \mathrm{~mm}$, and an effective focal length of $326.7 \mathrm{~mm}$ (some of the terms used with OAP's are explained in Fig. 2). The chalcogenide glass infrared (CIR) fiber has a core diameter of $1 \mathrm{~mm}$ and a numerical aperture of 0.37 . The fiber aperture serves as the field stop of the system and the magnification is unity, so that the spot size viewed on the sample is nominally $1 \mathrm{~mm}$ in diameter. However, in order to make the design more compact, as required by the application for which it was developed, the parabolic mirrors are set up at an off-axis angle of $35^{\circ}$ instead of $30^{\circ}$, and the spot size on the sample is expected to be somewhat larger.

The use of reflective instead of refractive optics offers some distinct advantages. The absence of chromatic aberrations ensures that the size of the spot on the sample from which radiance is collected is the same for all pyrometer channels. In addition, it facilitates alignment with visible light. Finally, the cost of diamond-turned metallic mirrors is significantly lower than infrared lenses of the same size, especially when a broadband anti-reflective (BBAR) coating is required.

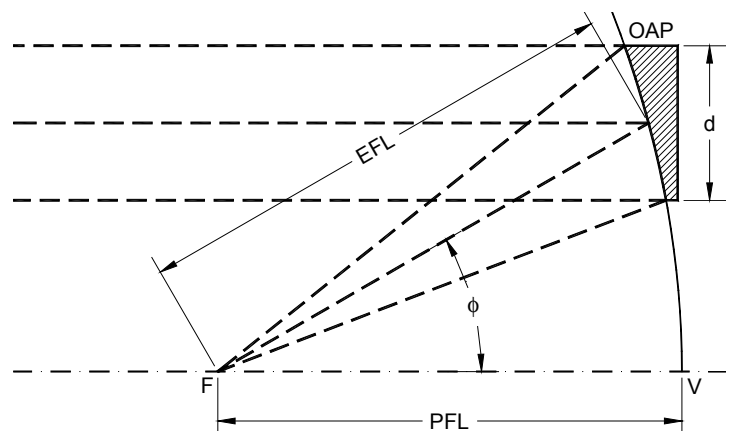

FIGURE 2. Schematic of an off-axis parabolic (OAP) mirror. $\mathrm{F}$, focal point; $\mathrm{V}$, vertex of the parent parabola; PFL, focal length of the parent parabola; EFL, effective focal length; $\phi$, off-axis angle; $d$, diameter of the OAP.
Although the surface quality of diamond-turned mirrors is not as high as that of polished ones, the additional scatter that is introduced might be a problem only at short wavelengths (visible and ultraviolet).

The use of aspheric optics (instead of concave mirrors, for instance) allows the design of faster (low f-number) systems while keeping the aberrations small. The use of off-axis instead of on-axis parabolas eliminates the problem of central obscuration typical of Cassegrain designs.

\section{Detector Unit}

Figure 3 shows the optical arrangement on the detector unit. A lens is used to collimate the light from the CIR fiber. Three dichroic beam splitters spectrally divide the collimated beam in four for detection by an equal number of photodiodes. Broad bandwidth interference filters further define the spectral ranges of the four channels and enhance blocking of unwanted wavelengths. A lens in front of each detector focuses the beam on the photodiode.

All lenses are zinc selenide ( $\mathrm{ZnSe})$ meniscus lenses, $25.4 \mathrm{~mm}$ in diameter, with a focal length of $25.4 \mathrm{~mm}$ and a BBAR-coating on both sides that is effective from 1 to $8 \mu \mathrm{m}$. Essentially, the five lenses form four doublets in the conventional way, with the wavelength-selecting elements in the collimated portion of the beam, that image the fiber on the photodiodes. The collimating lens is common to all doublets.

The most important components on this detector unit are the dichroic beam splitters. They were commercially designed and fabricated specially for this pyrometer to operate as long-pass filters in transmission and as short-pass filters in reflection, at a $45^{\circ}$ angle of incidence. Their reflectance and

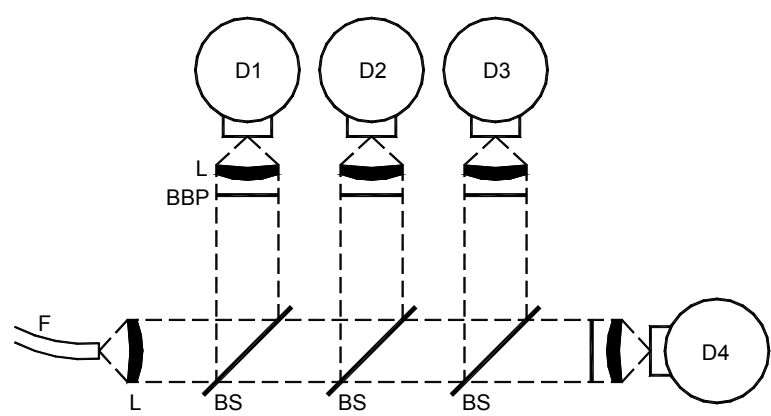

FIGURE 3. Schematic diagram of the detector unit of the four-wavelength infrared pyrometer. F, fiber; L, BBARcoated ZnSe lenses; BS, dichroic beam splitters; D1-D4, $\mathrm{LN}_{2}$-cooled InSb detectors; BBP, broad bandpass filters. 
transmittance in the wavelength range of interest, specified as 1.3 to $6 \mu \mathrm{m}$, are both $90 \%$ or higher. They are $50.8 \mathrm{~mm}$ in diameter, $3 \mathrm{~mm}$ thick, with the dichroic coating on the front surface (toward the incoming beam) and a BBAR-coating on the back surface of the ZnSe substrate. They are arranged in order of increasing transmitted wavelength. Specifically, the first dichroic beam splitter (closest to the incoming light) is an LP2100, which means that it transmits wavelengths above $2100 \mathrm{~nm}$ and reflects the shorter wavelengths. The second in order is an LP2700 and the third one is an LP4400. Accordingly, detector D1 in Fig. 3 sees the shortest wavelengths, whereas D4 sees the longest.

Indium antimonide ( $\mathrm{InSb}$ ) photodiodes, $1 \mathrm{~mm}$ in diameter, are used to detect the radiance in the four channels. They are placed in side-viewing dewars with $1 \mathrm{~mm}$ thick, AR-coated sapphire windows and are cooled with liquid nitrogen $\left(\mathrm{LN}_{2}\right)$ to $77 \mathrm{~K}$. A cold aperture inside the dewar limits their field of view to $60^{\circ}$ (full angle) to restrict unwanted background radiation. The photodiodes were specially selected to be compatible with custom-made, low-noise, $20 \mathrm{MHz}$ bandwidth pre-amplifiers.

The entire detector unit can be placed in a steel enclosure with radio-frequency (RF) gaskets for EMI shielding, when needed. In that case, the detectors are powered by four rechargeable lead-acid batteries (gelcells) located inside the same enclosure.

\section{CHARACTERISTICS AND PERFORMANCE}

\section{Spectral Responsivity}

The combined spectral propagance ${ }^{1}$ and responsivity of the four pyrometer channels was measured from 500 to $6000 \mathrm{~nm}$ in $20 \mathrm{~nm}$ steps. The measurement was performed with the CIR fiber placed directly at the exit aperture of an automated monochromator system, using detectors with NISTtraceable calibration as a reference. The four spectral bands are shown, peak-normalized, in Fig. 4. Their centers of gravity ${ }^{2}$ are at $1.82 \mu \mathrm{m}, 2.39 \mu \mathrm{m}, 3.44 \mu \mathrm{m}$, and $5 \mu \mathrm{m}$. The respective full-width-half-maximum (FWHM) bandwidths are $298 \mathrm{~nm}, 393 \mathrm{~nm}, 536 \mathrm{~nm}$,

\footnotetext{
${ }^{1}$ The fraction of incident flux that is (successfully) propagated over an extended path [4]. It includes the attenuation due to all transmittances and reflectances over the entire path.

${ }^{2}$ Weighted averages of wavelength, with the relative responsivity as the weighting function.
}

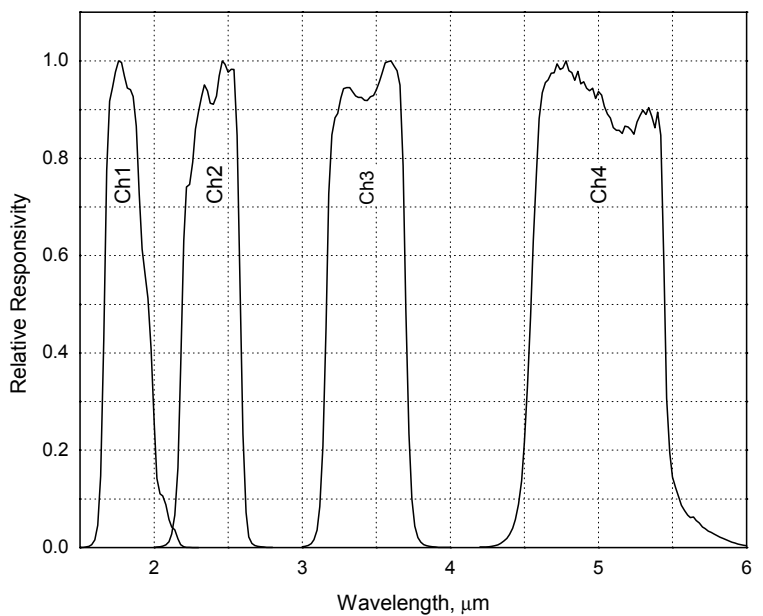

FIGURE 4. Peak-normalized responsivity of the four pyrometer channels.

and $903 \mathrm{~nm}$.

Each one of the pyrometer channels can be treated as a stand-alone finite-bandwidth pyrometer whose measurement equation, relating the output signal $\mathrm{S}$ (in units of $\mathrm{V}$ ) to the radiance temperature $\mathrm{T}$ of the target, is approximated by

$$
S(T)=b \Theta \tau^{*}(\lambda)_{\max } \int_{\Delta \lambda} \tau^{*}(\lambda)_{p . n .} L_{\lambda, b b}(\lambda, T) d \lambda
$$

In the above equation, $b$ (in units of $\mathrm{V} \cdot \mathrm{A}^{-1}$ ) is a constant determined by the electronic conversion of the photodiode current into a proportionate voltage, $\Delta \lambda$ is the wavelength interval over which the instrument responsivity is significantly non-zero, and $L_{\lambda, b b}(\lambda, T)$ is the blackbody spectral radiance as a function of wavelength $\lambda$ and blackbody temperature T. $\tau^{*}(\lambda)$ stands for

$$
\tau^{*}(\lambda)=\tau(\lambda) R_{\Phi}(\lambda)
$$

where $\tau(\lambda)$ is the spectral propagance of the entire path from the sample to the detector and $R_{\Phi}(\lambda)$ is the spectral flux-responsivity (in units of $\mathrm{A} \cdot \mathrm{W}^{-1}$ ) of the detector. $\tau^{*}(\lambda)_{\max }$ is the maximum value of $\tau^{*}(\lambda)$ with respect to which the latter is peak-normalized (p.n.) to yield the dimensionless quantity $\tau^{*}(\lambda)_{p . n .} . \quad \Theta$ is the throughput $^{3}$ of the beam that is collected by the front optics into the CIR fiber

$$
\Theta=\iint_{A} \cos \theta d \omega d A
$$

\footnotetext{
${ }^{3}$ This is also known as the geometric extent of the beam, and as its étendue.
} 
where $\mathrm{A}$ is the area of the fiber core and $\omega$ the solid angle subtended by the second parabolic mirror at the fiber entrance aperture.

The shape of the pyrometer signal $\mathrm{S}$ as a function of target radiance temperature $\mathrm{T}$ can be computed by evaluating just the integral in Eq. (1), without an accurate knowledge of the values of $b, \Theta$, and $\tau^{*}(\lambda)_{\max }$.

\section{Calibration}

A temperature calibration of the pyrometer was performed using a cavity blackbody. Figure 5 shows the measured signals as a function of blackbody temperature from 373 to $1173 \mathrm{~K}$.

The solid curves are computed using the integral in Eq. (1), as described in the previous section, and normalized with respect to the measured signals at the highest calibration temperature. A comparison of the computed signals with the measured ones shows good agreement, indicating that there were no significant errors in the measurement of the spectral responsivity.

Another way to check the quality of the spectral responsivity measurement is to plot the measured signals against the numerical value of the integral in Eq. (1). If $\tau^{*}(\lambda)_{p . n .}$, which includes the spectral fluxresponsivity of the detectors, is interpreted as an "effective" transmittance, then that integral can be treated as the blackbody radiance giving rise to a signal in each channel. Assuming the detectors are linear, the measured signals ought to be proportional to

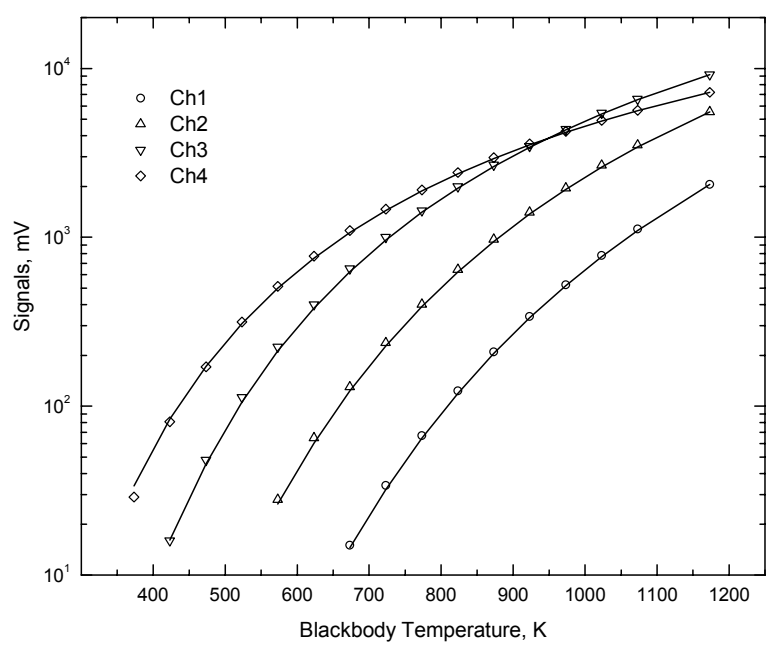

FIGURE 5. The four pyrometer signals as a function of blackbody temperature. The symbols represent the measured signals, whereas the solid curves have been computed using the measured spectral responsivities of the four channels.

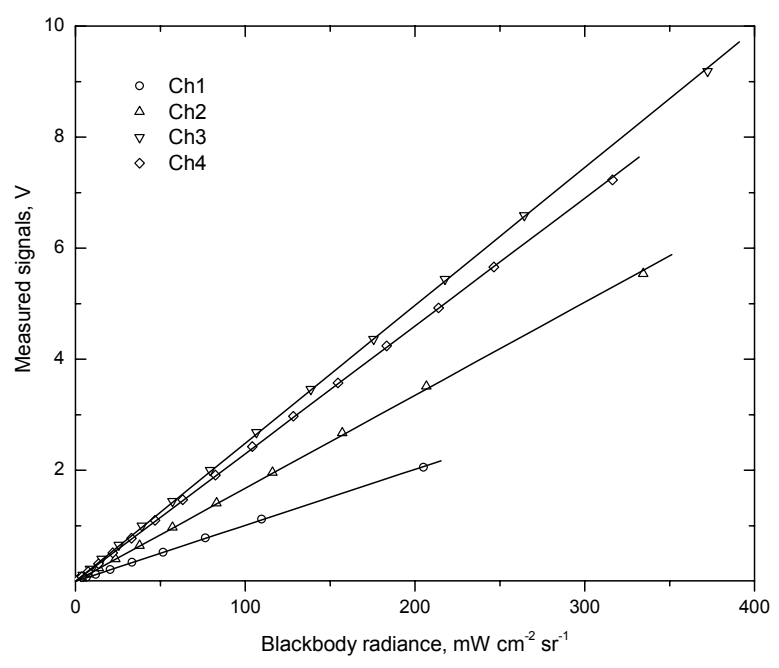

FIGURE 6. The four pyrometer signals as a function of blackbody radiance. The blackbody radiance incident on each detector was computed using the measured spectral responsivities of the four channels. The solid curves are linear fits that were forced through the origin.

this radiance. As can be seen in Fig. 6, there was indeed no significant deviation from linearity. The different slopes of the four straight lines can be attributed mainly to differences in $\tau^{*}(\lambda)_{\max }$, since $b$ and $\Theta$ should be close to identical for the four channels. Because the spectral flux-responsivity of $\mathrm{InSb}$ photodiodes at $77 \mathrm{~K}$ increases monotonically with wavelength between 1.5 and $5.2 \mu \mathrm{m}$ [5], one would also expect to see a monotonic increase in the slopes, going from $\mathrm{Ch} 1$ to $\mathrm{Ch} 4$. The fact that the linear fit corresponding to $\mathrm{Ch} 3$ has a higher slope than that of Ch4 in Fig. 6 can possibly be explained by the increased attenuation of the CIR fiber above $4.8 \mu \mathrm{m}$, according to the manufacturer, and/or by differences in the alignment of the focusing lenses in front of the third and fourth detector on the detector unit.

\section{DETERMINATION OF TEMPERATURE}

The method that will be used to analyze the pyrometry data is described in references 6 and 7. It seems to be well suited to shock experiments, when no simultaneous reflectance or emittance measurement is available.

The method is based on an assumed band of allowed emittance values for each pyrometer channel. In the absence of any other information, the upper bound is set to unity (blackbody). The lower bound is typically chosen just below the static emittance value 
at each wavelength for the specific sample (usually measured at room temperature). The main justification for this assumption is the fact that often during shock experiments the surface quality of the sample deteriorates, causing an increase in emittance. These emittance bands then yield an equal number of allowed temperature bands. Since it is further assumed that a single thermodynamic temperature produces the measured radiances in all pyrometer channels, the range of possible temperatures is confined to the intersection of these temperature bands. The most precise temperature measurements using this technique are obtained when the lower bounds on the dynamic emittance are strongly wavelength dependent.

Once a range of possible temperatures has been determined, values for the emittance in each pyrometer channel can be computed. These emittances can be a helpful indicator that large optical backgrounds, such as light originating from the use of $\mathrm{HE}$, were present during the measurement. In such cases the method will not result in a single temperature describing the measured radiances in all four spectral bands within the given emittance constraints.

\section{CONCLUSION}

A portable, high-speed, four-wavelength, infrared pyrometer for dynamic temperature measurements on shocked targets has been designed and constructed. It is capable of measuring blackbody temperatures in the nominal range of 400 to $1200 \mathrm{~K}$ with 20 ns-resolution. Its modular design offers great flexibility in the choice of front optics. The use of off-axis parabolic mirrors offers some distinct advantages over refractive, spherical optics in instruments that operate in the IR and span a large wavelength range.

Future plans include (i) the use of $90^{\circ}$ off-axis parabolic mirrors that will allow a more compact design, (ii) the use of a visible alignment laser instead of a viewing microscope for alignment purposes, (iii) the replacement of the sapphire windows in the detector dewars with AR-coated silicon windows that will increase the sensitivity at the longer wavelengths and at the same time block all of the visible background light. Existing $1 \mathrm{~mm}$-core IR fibers are at best difficult to work with and are continuously being improved. Problems include high fragility, poor transmission compared to fibers used in the visible range of the spectrum, and high reflection losses at the ends due to the high refractive index of the core material (on the order of 2.4). Therefore, the possibility of a fiberless pyrometer, where the light from the front optics is transmitted over moderate distances through air to the detector unit, is also being explored.

\section{REFERENCES}

1. Tenney, A. S., "Radiation Ratio Thermometry," in Theory and Practice of Radiation Thermometry, edited by D. P. DeWitt and G. D. Nutter, Wiley-Interscience, New York, 1988, pp. 459-494.

2. Nordine, P. C., High. Temp. Sci. 21, 97-109 (1986).

3. Neuer G., Fiessler L., Groll M., Schreiber E., "Critical Analysis of the different methods of multiwavelength pyrometry," in Temperature: Its Measurement and Control in Science and Industry, Vol. 6, edited by J. F. Schooley, American Institute of Physics, New York, 1992, pp. 787-790.

4. Nicodemus, F. E., Kostkowski, H. J., "Distribution of Optical Radiation with respect to Position and Direction" in Self-Study Manual on Optical Radiation Measurements, edited by F. E. Nicodemus, Nat. Bur. Stand. Tech. Note 910-1, 1976, p. 38.

5. Dereniak, E. L., Boreman, G. D., Infrared Detectors and Systems, Wiley-Interscience, New York, 1996, pp. 306307.

6. Partouche-Sebban, D., Holtkamp, D. B., Bartsch, R. R., Lee, H., Schmitt, G. G., Rev. Sci. Instrum. 72, 3008-3012 (2001).

7. Partouche-Sebban, D., Holtkamp, D. B., Pélissier, J. L., Taboury, J., Rouyer, A., Shock Waves 11, 385-392 (2002). 